\title{
Anatomy of Brachial Plexus Above The Clavicle
}

\author{
Shivaprakash $S^{1}$, Georg Feigl ${ }^{2}$, Sandeep M. Diwan ${ }^{3}$ \\ ${ }^{1}$ Department of Anatomy, JSS Medical College, JSS Academy of Higher Education and Research, Mysore, Karnataka, India. \\ ${ }^{2}$ Private Universitat Witten/Herdecke gGmbH Alfred-Herrhausen-StraBe 50, D-58448 Witten. \\ ${ }^{3}$ Department of Anaesthesia, Sancheti Hospital, Pune, Maharashtra, India.
}

\section{Introduction}

The neck is a compact structure which hosts the aero-digestive and neurovascular structures. Nerve roots arising from the spinal cord form an important network of nerves the 'Brachial Plexus (BP)' that innervates the upper limb and lies partly in the posterior triangle of neck and partly in the axilla. The BP is complex matrix sandwiched between muscles proximally and muscles and vessels distally at and above the level of clavicle. It consists of roots, trunks, cords \& branches (Figure 1). Roots and trunks are supraclavicular, divisions are retro clavicular, cords and their branches are infraclavicular. The position of the plexus relative to the clavicle varies, it is higher in the erect position and lower when recumbent [1]. It is broad and presents little of a plexiform arrangement at its commencement, is narrow opposite the clavicle, divides opposite the coracoid process into numerous branches and becomes broad and forms a denser interlacement in the axilla [2]. Brachial plexus is formed by the ventral rami of lower four cervical nerves and the first thoracic spinal nerves with variable contribution (slender twigs) from the fourth cervical and second thoracic nerve.

\section{Arrangement of BP}

The upper limb is mainly innervated by the brachial plexus, is formed by the ventral rami of spinal nerves of the spinal cord segments C5 to T1 [3]. Auxiliary fibres may from C4 (pre-fixed) or T2, 3 (post-fixed).

The origin of BP is from C5, 6, 7,8 and T1. The C5 and C6 unite to form the superior trunk (ST), the C7 continues as the middle trunk and the C8 and T1 merge to form the inferior trunk (IT) (Figure 1). The trunks further divide into anterior and posterior divisions below the omohyoid and above the clavicle.

Along its course from proximal (medial) to distal (lateral), the brachial plexus is represented in four areas: interscalene, posterior triangle of neck (supraclavicular), infraclavicular fossa, and axillary fossa [4]. Nerve blocks are placed according to the following areas: Interscalene, Supraclavicular, Infraclavicular and Axillary.

\section{The course of the BP}

The anterior primary rami exit the intervertebral foramina (IVF) through the sulcus formed by the anterior and posterior tubercles of the corresponding transverse processes of the cervical vertebrae. Anterior primary rami courses over Costo-tranverse bar (Figure 2) [Costo-transverse bar connects anterior and posterior tubercles of TP, Scalenus anterior and Scalenus medius is attached to anterior \& posterior tubercles respectively]. The trunk of cervical spinal nerve after emerging from IVF divides into ventral \& dorsal rami, dorsal rami curves backwards around the articular pillars and supplies para vertebral muscles while ventral rami passes over costotransverse bar and emerges between Anterior \& Middle scalene muscles.

After leaving the transverse process, the roots of the plexus descend in front of the middle scalene muscle (figure 3 ), which arises from the posterior tubercles of the transverse processes of the lower six cervical vertebrae. The insertion of this muscle on the first rib is separated from that of the anterior scalene by the subclavian groove, through which the artery and inferior trunk of the plexus will pass (Figure 1). The anterior scalene muscle arises from the anterior tubercles of the transverse processes of the $3^{\text {rd }}, 4^{\text {th }}, 5^{\text {th }}$, and $6^{\text {th }}$ cervical vertebrae and inserts on the scalene tubercle of Address of Correspondence: Dr. Sandeep Diwan, Department of Anaesthesia, Sancheti Hospital, Pune, Maharashtra, India.

E-mail: sdiwan14@gmail.com

DOI: 10.13107/ijra.2021.v02i01.023 | (C) 2021 International Journal of Regional Anaesthesia | Available on www.ijrajournal.com | This is an Open Access article distributed under the terms of the Creative Commons Attribution Non-Commercial License (http://creativecommons.org/licenses/by-nc/3.0) which permits unrestricted non-commercial use, distribution, and reproduction in any medium, provided the original work is properly cited.

How to cite this article: Shivaprakash S, Feigl G, Diwan SM | Anatomy of Brachial Plexus Above The Clavicle | International Journal of Regional Anaesthesia | January-June 2021; 2(1): 29-34. 
the first rib, thus separating the subclavian vein from the subclavian artery, which lies posterior to this insertion (Figure 1). At the first rib, the BP in the form of divisions are horizontally situated between the SCA medially and the MSMlaterally (Figure 1).

\section{The Interscalene Area}

The trunks of BP emerge between the ASM and MSM (Figure 3 ) and course from cephalad to caudal and medial to lateral. The BP invaginates the prevertebral fascia along with it, which forms a tubular extension around the BP. The Phrenic nerve (PN) lies on the anterior surface of the ASM and courses from lateral to medial (Figure 3 ).

\section{Boundaries}

The boundaries of the interscalene area, and the arrangement of the trunks, are illustrated by a cross-section made at the level of C6, (Figure 3), showing the nerve fascicles of trunks between the posterior fascia of the anterior scalene muscle and the anterior fascia of the middle scalene muscle. The neural elements (dark brown) are interspersed with light brown connective tissue (Figure 4). The connective tissue forms a protective barrier to the neural elements to the advancing needle tip.

\section{The Interscalene Sub-areas (Figure 5),}

Feigl [5] notified two anatomically distinct spaces are contained in the interscalene gap:

The lateral prevertebral space (Figure 5) bounded laterally by the anterior scalene muscle and medially by the long muscle of the neck, connects the prevertebral space to a ventrally located space without the prevertebral fascia. Injections of large volumes of local anaesthetic agents in this space might spread in medial directions inside a space filled with loose connective tissue, thus potentially leading to blockade of the recurrent laryngeal nerve, the sympathetic chain, or autonomic innervation systems of the heart.

The medial scaleno-vertebral triangle (SVT) (Figure 5), is bounded medially by the lateral edge of the longus colli muscle, laterally by the medial edge of the scalene anterior muscle and its base is formed by the first part of the subclavian artery (SA) [6]. Needle positioning in the SVT is unlikely under ultrasound but can lead to inadvertent arterialinjection.

\section{The Prevertebral Fascia and BP}

The prevertebral fascia (PVF) splits open to engulf the ASM and the MSM's. As the cervical roots emerge from the IVF they pierce the PVF and descend lateral to the ASM overlying the MSM (Figure 6). The trunks of the BP are invested/engulfed with the prevertebral fascia as they course caudal and lateral (Figure 6).

\section{Arrangement of Trunks of BP}

The superior trunk is visualized sandwiched between the ASM and the MSM's (Figure 3). The trunks as they are positioned vertical one over the other are designated as superior, middle, and inferior trunks (Figure 7). The PVF has been removed and demonstrates the external jugular vein and the transverse cervical artery with the $\mathrm{BP}$ in close approximation(Figure 6). As they descend, they track beneath the omohyoid muscle (Figure 1,2).

\section{The Phrenic Nerve}

The phrenic nerve is situated on the anterior surface of the ASM (Figure 3) beneath its fascia and descend from lateral to medial into the thoracic cavity. At times, the PN can be indistinguishable from the cervical root (Figure 8).

Blocks administered high in the interscalene area leads to phrenic nerve palsy.

\section{Supraclavicular Area}

At and beneath the omohyoid the trunks ramify into divisions and form the supraclavicular brachial plexus in the 'supraclavicular area'. Alternatively this is referred to as the 'posterior triangle of neck'. [The posterior triangle is a wider area extending between the mastoid process and clavicle. The inferior belly of omohyoid divides the posterior triangle into larger occipital triangle, and a lower Subclavian triangle (Supraclavicular triangle)]. The ventral branches of C5/C6 continuing as superior, $\mathrm{C} 7$ as middle, and $\mathrm{C} 8 / \mathrm{T} 1$ as inferior trunk.

\section{Boundaries of Supraclavicular BP}

The posterior triangle of the neck is bounded anteriorly (or medially) by the posterior border of the sternocleidomastoid muscle, posteriorly (or laterally) by the anterior border of the trapezius muscle, and caudally by the clavicle (Figure 9).

\section{Arrangement of Divisions}

The superior and middle trunks is cranial and lateral to the subclavian artery. The inferior trunk can be found dorsal to the artery where the "corner pocket" is located (Figure 10). The superior and middle trunks may be separated from the inferior trunk at the level of subclavican artery (Zuckerkandl-Sebileau ligaments) [5].

The divisions emerging from the trunks of the plexus cross the first rib, they are joined by the subclavian artery, which lies in a plane anterior to the trunks, so that the inferior trunk lies behind the artery in the subclavian groove with the middle and superior trunks above the level of the vessel 


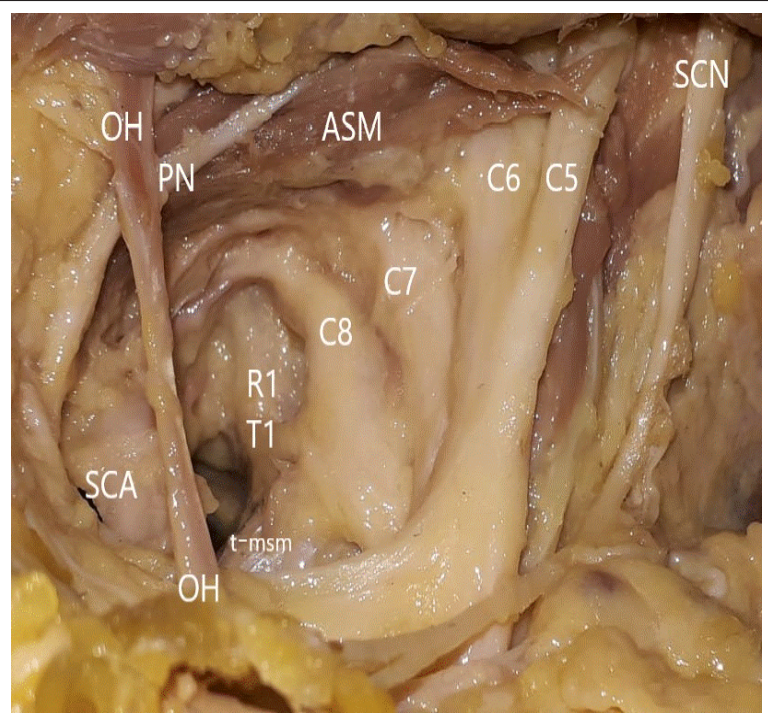

Figure 1: The 'Brachial Well' - The interscalene space (gap)is widened with the ASM and the MSM retracted medial and lateral, respectively. The posterior and lateral brim of the well is formed by the $\mathrm{C} 5$ and $\mathrm{C} 6$ anastomosing into the superior trunk. Along the brim the foot-steps ate the $\mathrm{C} 5, \mathrm{C} 6, \mathrm{C} 7, \mathrm{C} 8$ and the $\mathrm{R} 1$ (first rib). The anterior of the well is walled off by the omohyoid $(\mathrm{OH})$ muscle. The shiny tendon of the MSM ( $\mathrm{t}$ msm) is attached to the R1. The C8 unites with $\mathrm{T} 1$ to form the inferior trunk. The (SCA) appears beneath the $\mathrm{OH}$ muscle. Shivaprakash S; Sandeep Diwan; Department of Anatomy, JSSMC, Mysore

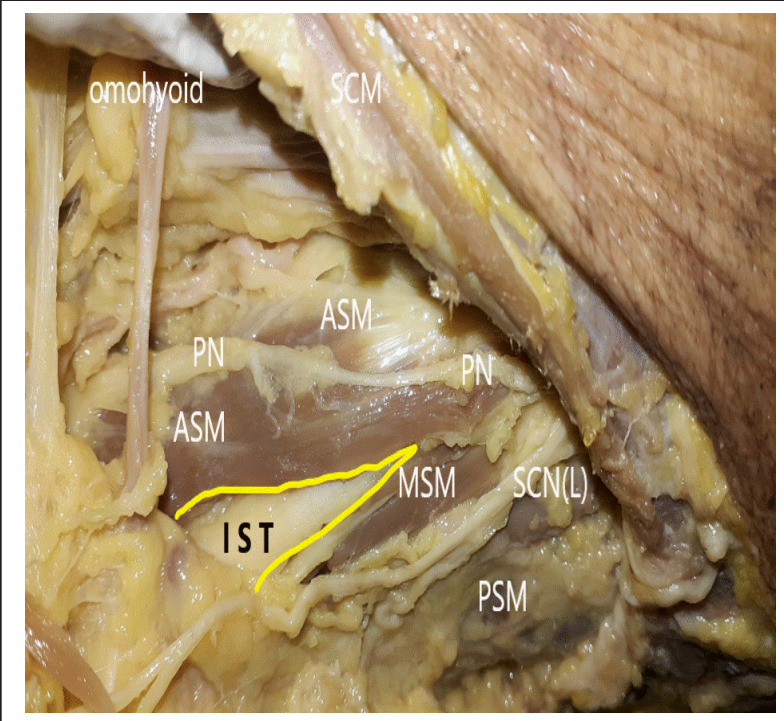

Figure 3: Left brachial dissection revealing the emergence of interscalene trunks (IST) between the anterior scalene muscle (ASM) medially and the middle scalene muscle laterally. The phrenic nerve (PN) courses from lateral to medial on the ASM, deep to its sheath. The supraclavicular nerve arises from a higher side courses laterally and caudal over the MSM and the posterior scalene muscle (PSM): Shivaprakash S ; Sandeep Diwan; Department of Anatomy, JSSMC, Mysore

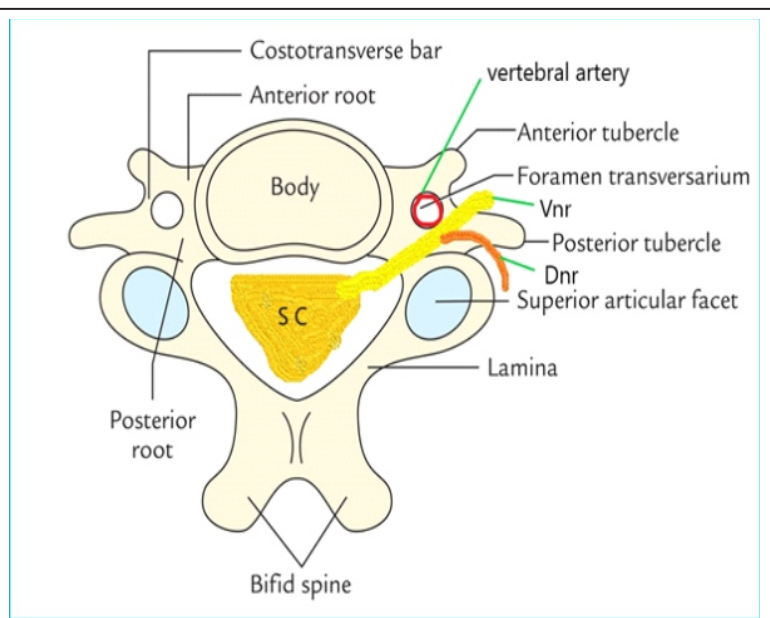

Figure 2 : Costotransverse bar and relation of spinal root costal margin

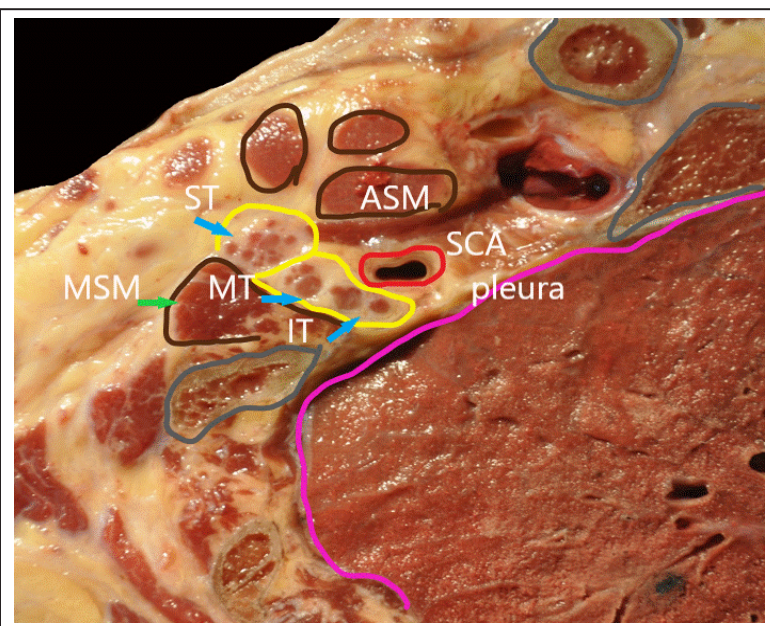

Figure 4: Axial section at the level of T1-2 depicting the outlay of the brachial plexus and its relation to the pleura and the subclavian artery (SCA). MSM - middle scalene muscle; STsuperior trunk; MT-middle trunk; IT- inferior trunk; SCAsubclavian artery; ASM- anterior scalene muscle: Georg Feigl ; Sandeep Diwan; Anatomy Graz

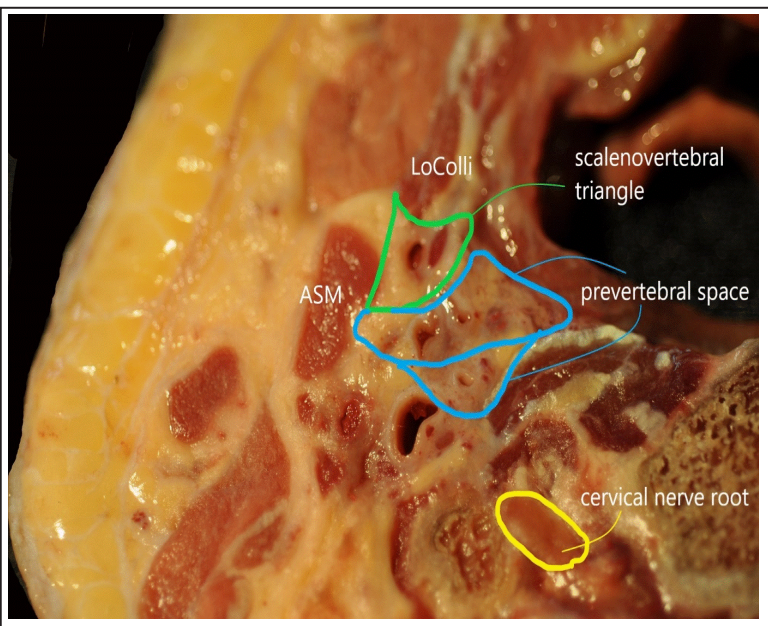

Figure 5: The two triangles described in the interscalene area : the scalenovertebral triangle (green) and the prevertebral space (blue) ;ASM -anterior scalene muscle; LoColli - longus colli Georg Feigl ; Sandeep Diwan; Anatomy Graz 


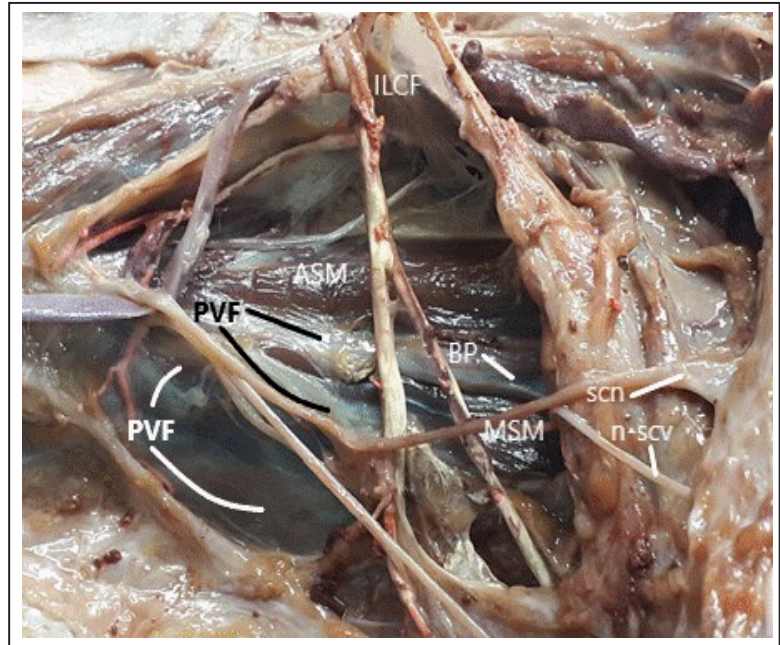

Figure 6: The right prevertebral fascia (PVF) engulfing the BP, and the scalene muscles. n-scv: nerve to subclavius; scnsupraclavicular nerve: Georg Feigl ; Sandeep Diwan; Anatomy Graz

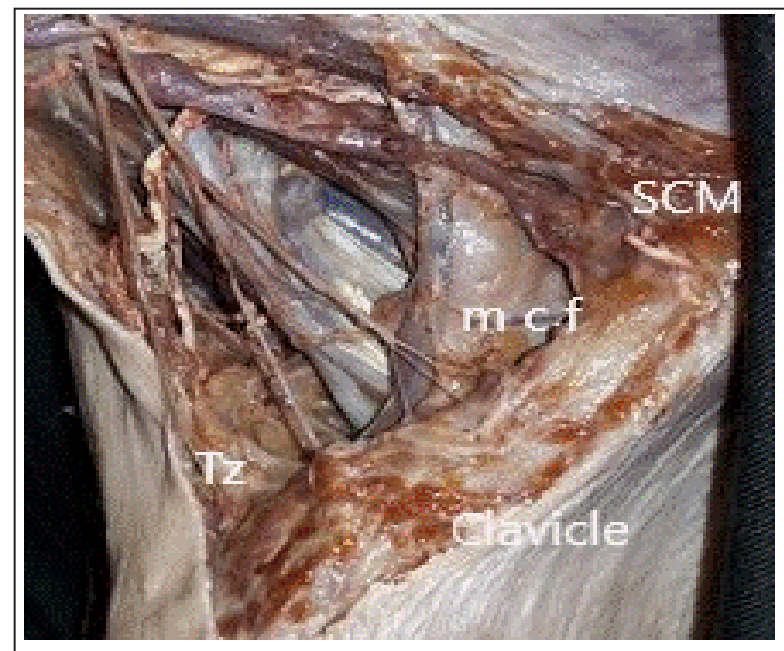

Figure 9: Boundaries of the right supraclavicular BP SCM -sternocleidomastoid; Tz-Trapezius; $\mathrm{m} \mathrm{c} \mathrm{f}$ - middle cervical fascia

Georg Feigl ; Sandeep Diwan; Anatomy Graz

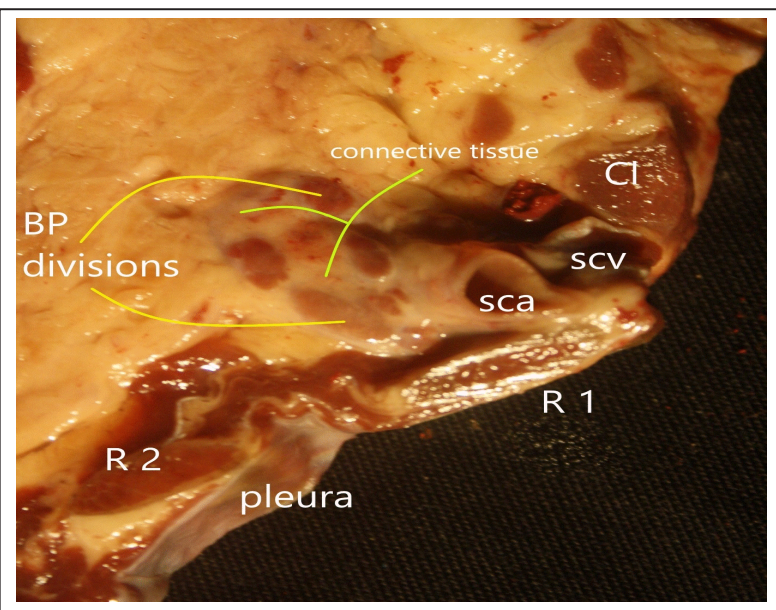

Figure 10: Coronal section at the level of first rib (R1). Georg Feigl ; Sandeep Diwan; Anatomy Graz

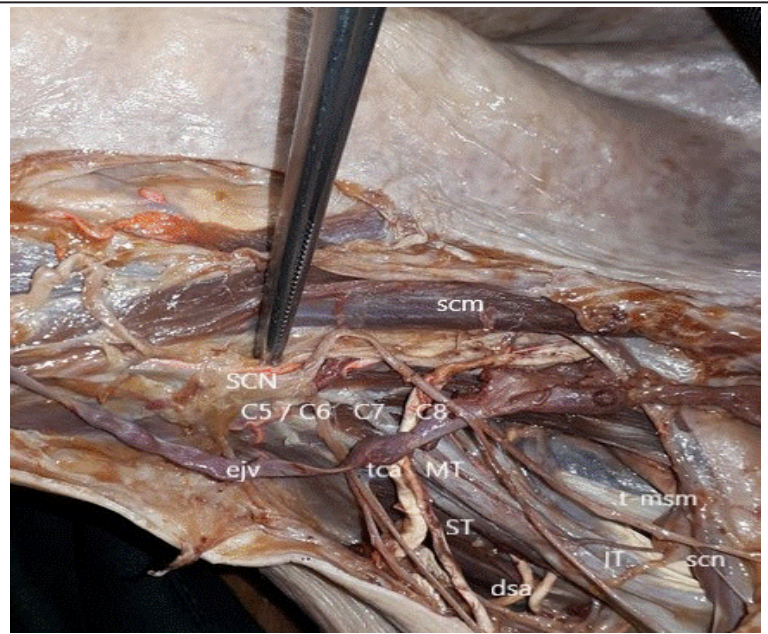

Figure 7: Right BP dissection : SCN -supraclavicular nerve origin; C5-8 are the roots emerging from their respective costotransverse bars. They form the superior (ST), middle (MT) and inferior trunks (IT) respectively. ejv-external jugular vein; tcatransverse cervical artery; dsa-dorsal scapular artery; scnsupraclavicular nerve; t-msm:tendon of MSM; scmsternocleidomastoid: Georg Feigl ; Sandeep Diwan; Anatomy Graz

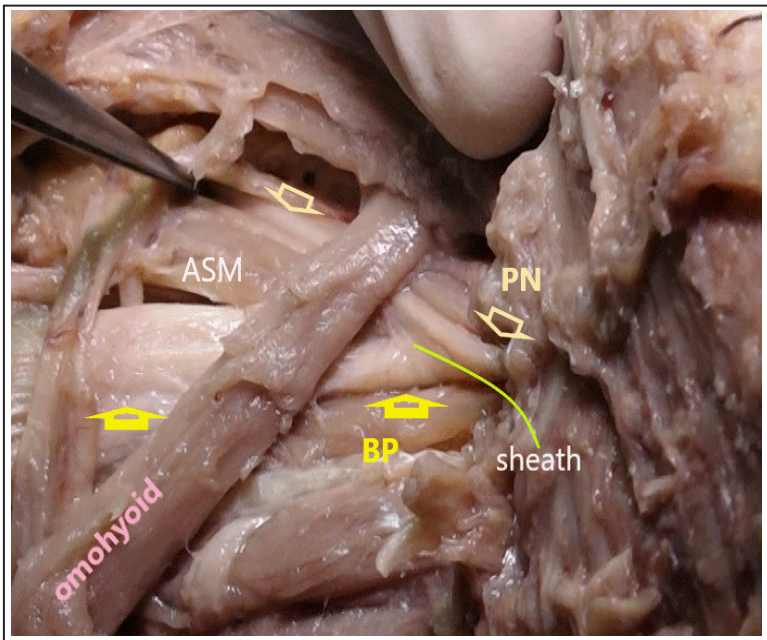

Figure 8: Left side brachial plexus dissection : Depicting the close association of phrenic nerve and the BP roots engulfed in single sheath. The PN course lateral to medial on the anterior scalene muscle (ASM).

The Left Phrenic nerve looks like as if it arises from the superior trunk at the level of C6, engulfed by a sheath. The cadaveric dissection reveals the phrenic nerve (light pink) coursing over the anterior scalene muscle. The sheath (green line) engulfs the $\mathrm{PN}$ as well as the cervical root. The higher the interscalene block the higher the incidence of phrenic nerve block. Sandeep Diwan 


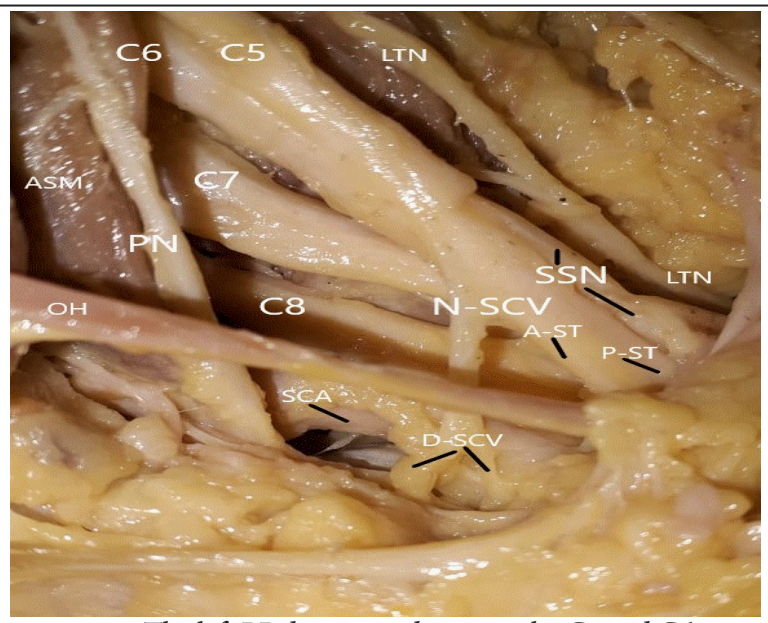

Figure 11: The left BP dissection depicting the C5 and C6 forming the superior trunk which gives off the suprascapular nerve (SSN), the anterior and posterior division of the superior trunks (A-ST and P-ST) and the nerve to subclavius ( N-SCV). The N-SCV divide into branches (D-SCV). Roots emerging from $\mathrm{C} 6$ and $\mathrm{C} 7$, forms the lateral thoracic nerve (LTN).The C7 continues as the middle trunk while the $\mathrm{C} 8$ unites with $\mathrm{T} 1$ (not seen) at the level of subclavian artery to form the inferior trunk[ (IT) - not seen]. The phrenic nerve runs on the anterior scalene muscle (ASM). Sandeep Diwan ; Shivaprakash S ; Department of Anatomy, JSSMC

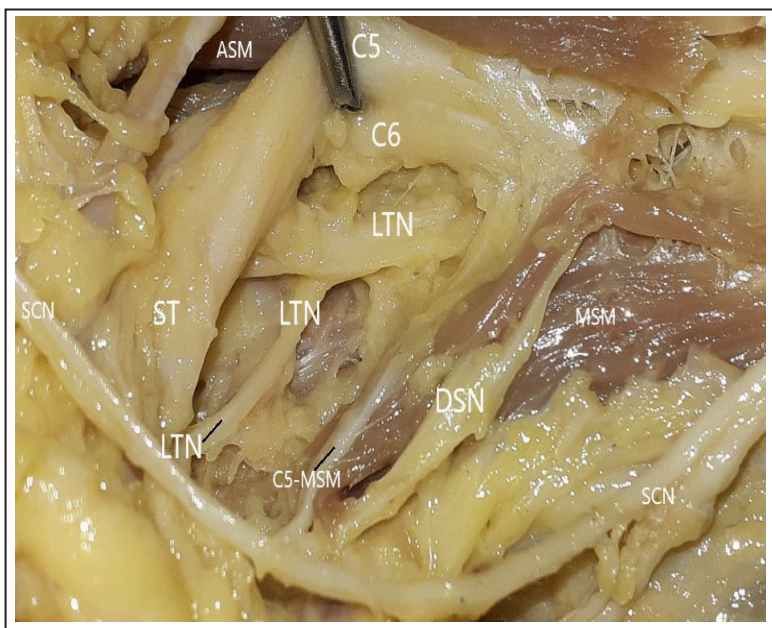

Figure 13: Left Brachial Dissection

Retracting the cervical roots medially, the roots emerging from C5.6 and 7 form the long thoracic nerves it courses in front the middle scalene muscle and behind the BP. A branch from $\mathrm{C5}$ also innervates the middle scale muscle (C5-msm)

Shivaprakash S ; Sandeep Diwan; Department of Anatomy JSSMC

Figure 15: Cross-section at the level of Transverse section at T1 demonstrating the internal thoracic artery (ITA) and the thyrocervical trunk (TCT) as they emerge from the subclavian artery (SCA). TP-transverse process;ar-T1=anterior ramus of T1; t-T1- trunk of T1; SC-spinal cord. Sandeep Diwan; Shivaprakash S ; Department of Anatomy JSSMC

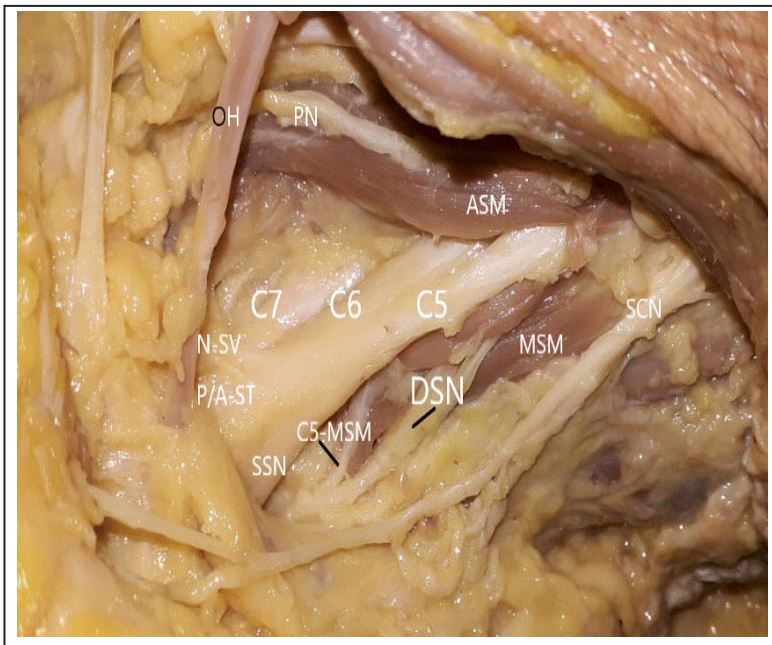

Figure 12: Left Brachial Plexus Dissection

The sternocleidomastoid (SCM) is retracted laterally. The phrenic nerve $(\mathrm{PN})$ is situated on the anterior scalene muscle (ASM). Separation of the ASM and the middle scalene muscle (MSM) reveals the $\mathrm{C} 5$ root emerging between the slips of ASM, followed by the C6 root beneath the ASM at its lateral border. The $\mathrm{C} 6$ join to form the superior trunk. The $\mathrm{C} 7$ is beneath and medial to the superior trunk. Between the muscular slips of the MSM are the roots arising from C6 and C7 which form the long thoracic nerve. The supraclavicular nerve (SCN) arise lateral and beneath the SCM. Shivaprakash S ; Sandeep Diwan; Department of Anatomy JSSMC

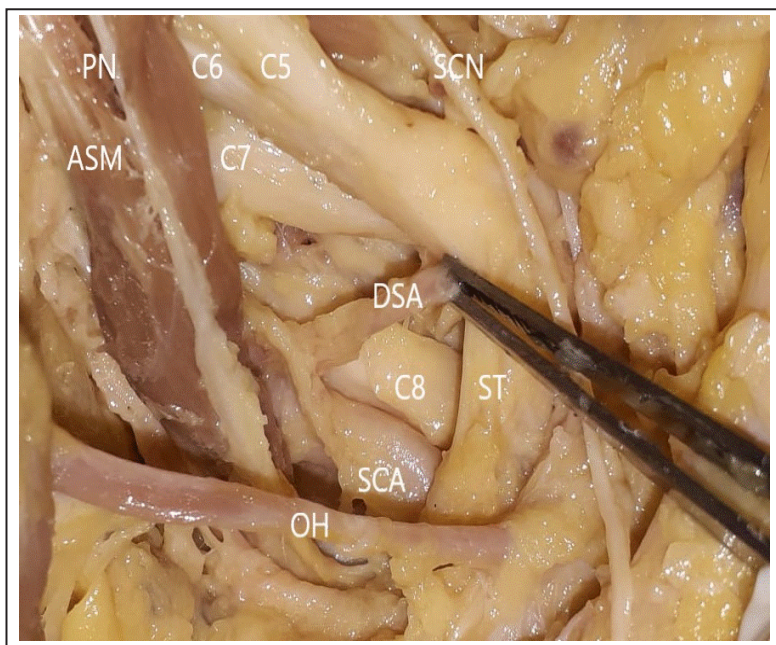

Figure 14: The Dorsal scapular artery emerging from the subclavian artery and passes between the $\mathrm{C} 7$ and $\mathrm{C} 8$ as predicted. Shivaprakash S ; Sandeep Diwan; Department of Anatomy, JSSMC

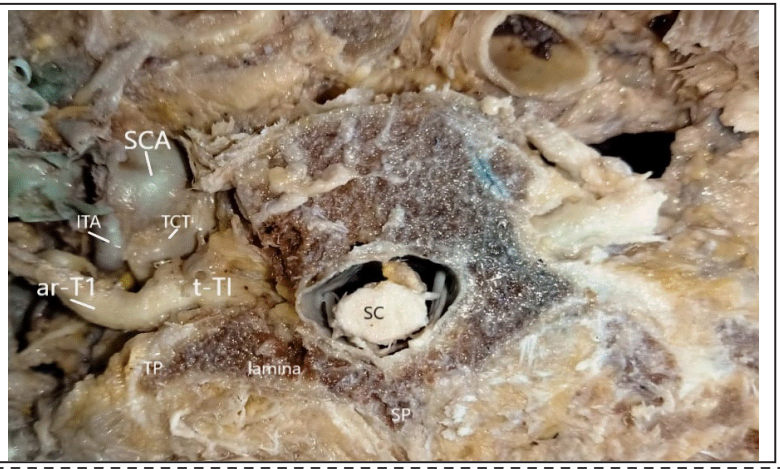

33 | International Journal of Regional Anaesthesia | Volume 2 | Issue 1 | January-June 2021 | Page 29-34 
(Figure 10,11).It is at this level that the artery and trunks, in moving laterally across the rib, invaginate the scalene fascia to form the "subclavian perivascular space" (SCPVS). The SCPVS is continuous with the interscalene space superomedially and the axillary perivascular space infero-laterally.

\section{Divisions and Branches}

Somewhere beneath the omohyoid, the trunks form divisions. In particular of note is the superior trunk (formed from C5 and C6) trifurcating into lateral most suprascapular nerve, the middle posterior division and the medial most anterior division labelled as 'SPA' (Figure 11). The C7 continues as the middle trunk and the $\mathrm{C} 8$ and $\mathrm{T} 1 \mathrm{form}$ the inferior trunk. All the trunks divide into anterior and posterior divisions. The subclavian nerve has its origin cranial to the clavicle and spreads out in a dorsal direction to innervate the subclavius muscle and to supply the periosteum of the medial and middle part of the clavicle, lateral to the insertion area of the coracoclavicular ligament.

\section{Nerves in the MSM}

Dissection of the MSM reveals the long thoracic and the dorsal scapular nerves. These nerves have their origin in the ventral rami of $\mathrm{C} 5$ to $\mathrm{C} 7$ (C8 being included in $8 \%$ of cases). After piercing the MSM the DSN nerves they appear at its lateral border and course on the dorsal to the brachial plexus and innervate muscles of the lateral and dorsal thoracic wall (Figure 12,13). At this point the long thoracic nerve is not visualized until the $\mathrm{C} 5$ and $\mathrm{C} 6$ roots are lifted to demonstrate the roots of $\mathrm{C} 5$ and $\mathrm{C} 6$ form the long thoracic nerve (Figure 12, 13). A branch of C5 innervating the middle scalene muscle is also seen.

\section{Vascular Structures in Posterior Triangle Of Neck}

The dorsal scapular artery [(DSA) (Figure 14) ] typically arises from either of two main sources: the subclavian artery (about $75 \%$ of all sides) or the transverse cervical artery (about $25 \%$ of all sides). DSA emerging directly from the subclavian artery passed between the upper and middle or middle and lower trunks of the brachial plexus (Figure 14). In the case of transverse cervical artery origin, the dorsal scapular artery arose as a branch that passed deep to the levator scapulae muscle before coursing to the rhomboid muscles [7]. An ultrasound study mentions the rate of visibility of the DSA at 34.0\% [8] though anatomical studies report that nearly $70 \%$ of DSAs arise from the subclavian artery, and in the remaining $30 \%$, the DSA may originate from the TCA [7]. The TCA coursed superficial to the scalene muscles and brachial plexus in at least $80 \%$ of scans [8].

A cross-section at the level of $\mathrm{C} 8$ and $\mathrm{T} 1$ vertebral body demonstrates the the trunk of the T1 (t-T1) in the intervertebral foramina goes on to form the anterior ramus of $\mathrm{T} 1$ ( $\mathrm{ar}-\mathrm{T} 1)$. In front of the $\mathrm{T} 1$ are the vascular structures (Figure 15).

\section{References}

1. G.J.Romanes. Cunningham's Manual of Practical Anatomy, vol 3. 15th ed.Oxford.Oxford university press;2014.Side of the Neck; 26-8.

2. Henry Gray F.R.S. Gray's Anatomy: Descriptive and Surgical. London. Parragon . book;2001( Reprint). Brachial plexus; 521-2.

3. Lanz T, Wachsmuth W. Praktische Anatomie. Berlin, Heidelberg, New York: Springer, 2004.

4. Hafferl A. Lehrbuch der topographischen Anatomie. Berlin, Heidelberg, New York: Springer, 1969

5. Feigl GC, Litz RJ, Marhofer P. Reg Anesth Pain Med 1-8; doi:10.1136/rapm-2020101435
6. Singal , T. Gupta, D. Sahni, A. Aggarwal Anatomy of scalenovertebral triangle: A vade mecum for clinicians Anatomie du triangle scalénovertébral : un vade mecum pour les cliniciens A. Morphologie $2020104174-181$

7. Reiner A, Kasser R. Relative frequency of a subclavian vs. a transverse cervical origin for the dorsal scapular artery in humans. Anat Rec 1996;244:265-8.

8. Murata, Hiroaki Sakai, Akiko ; Hadzic, Admir; Sumikawa, Koji The Presence of Transverse Cervical and Dorsal Scapular Arteries at Three Ultrasound Probe Positions Commonly Used in Supraclavicular Brachial Plexus Blockade; Anesthesia \& Analgesia: August 2012 - Volume 115 - Issue 2 - p 470-473.
Conflict of Interest: Nil

Source of Support: None 